\title{
Saccadic fatigability in the oculomotor system
}

\author{
J.A. Nij Bijvank (MD) $)^{a, b}$, L.J. van Rijn (MD, PhD) ${ }^{b, c}$, M. Kamminga ${ }^{d}$, H.S. Tan (MD, PhD) $)^{b}$ B.M.J. \\ Uitdehaag (MD, PhD) ${ }^{c}, A$. Petzold (MD, PhD, FRCP) $)^{\text {a,bee }}$, L.J. Balk (PhD) \\ ${ }^{a}$ Amsterdam UMC, Vrije Universiteit Amsterdam, department of Neurology, MS Center and Neuro-ophthalmology \\ Expertise Center, Neuroscience Amsterdam, de Boelelaan 1117, Amsterdam, The Netherlands \\ ${ }^{\mathrm{b}}$ Amsterdam UMC, Vrije Universiteit Amsterdam, department of Ophthalmology, Neuro-ophthalmology Expertise \\ Center, Neuroscience Amsterdam, de Boelelaan 1117, Amsterdam, The Netherlands. \\ 'Onze Lieve Vrouwe Gasthuis, department of Ophthalmology, Jan Tooropstraat 164, Amsterdam, The \\ Netherlands \\ ${ }^{\mathrm{d}}$ Technical Medicine, University of Twente, Drienerlolaan 5, Enschede, The Netherlands \\ eMoorfields Eye Hospital, The National Hospital for Neurology and Neurosurgery and the UCL Institute of \\ Neurology, London, United Kingdom
}

Corresponding author: J. Nij Bijvank, Amsterdam UMC, De Boelelaan 1118, 81 HZ Amsterdam Phone 020-4445063, j.niibijvank@vumc.nl

\section{Authors' disclosures}

J.A. Nij Bijvank: reports no disclosures, L.J. van Rijn: reports no disclosures, M. Kamminga: reports no disclosures, H.S. Tan: reports no disclosures, B.M.J Uitdehaag: has received consultancy fees from Biogen Idec, Genzyme, Merck Serono, Novartis, Roche and Teva, A. Petzold: member of the steering committee for the OCTiMS study (Novartis), no consulting fees. Performs OCT QC for the Passos study (Novartis), receives consulting fees. Dr Petzold is supported by the National Institute for Health Research (NIHR) Biomedical Research Centre based at Moorfields Eye Hospital National Health Service (NHS) Foundation Trust and University College London Institute of Ophthalmology, L.J. Balk: the Amsterdam MS Center received research support from TEVA . 


\section{Abstract}

Background: Fatigue is one of the most common and disabling symptoms in multiple sclerosis (MS), but challenging to quantify. This prospective study investigated if repeated saccadic eye movements enable measurement of oculomotor fatigability and can reflect on perceived fatigue in MS.

Methods: A standardized infrared oculography protocol (DEMoNS) was used for quantifying saccades in MS patients and healthy controls which included a first and a repeated pro-saccadic task (FPT and RPT). Saccadic peak velocity, latency, gain, area under the curve (AUC) and peak velocity divided by amplitude (Pv/Am) were calculated in both tasks. Perception based fatigue was assessed using the Checklist Individual Strength and the Neurological Fatigue Index (NFI). Linear regression models were used for assessing the relation between saccadic parameters and perceived fatigue.

Results: This study included 181 MS patients and 58 healthy controls subjects. From FPT to RPT, there were significant changes in saccadic parameters. Latency of both tasks was significantly related to $\mathrm{NFI}$ summary score (FPT: $\beta=0.022, p=0.049$, RPT: $\beta 0.023, p=0.021$ ). These relationships were weakened after adjustment for Expanded Disability Status score $(p>0.05)$. There was however no significant group difference in changes in saccadic parameters.

Conclusions: This study presents an objective and reproducible method for measuring saccadic fatigability. Saccadic fatigability was found to be of limited use in MS, and should be tested in conditions affecting ocular muscles or the neuromuscular junction.

Keywords: Multiple sclerosis, Eye movements, Saccades, Fatigue, Ocular Motility Disorders, Internuclear ophthalmoplegia. 


\section{Introduction}

Fatigue is one of the most disabling symptoms reported by multiple sclerosis (MS) patients, with prevalence rates reported in literature from $65 \%$ up to $>90 \%[1-4]$. The exact pathophysiological mechanisms of MS related fatigue are not clear. Relationships with clinimetrics are poor [5]. All this renders targeted treatment for fatigue challenging. One essential distinction made in fatigue measurement is between perception based fatigue and performance based fatigability, as emphasized in the clear taxonomy of fatigue by Kluger et al [6]. Perceived fatigue refers to the subjective assessment of fatigue sensation, which can have motor and cognitive features, most commonly selfreported by questionnaires. In contrast, performance fatigability refers to objective changes in performance of a task over a given time. This occurs during a prolonged motor or cognitive task, designed to induce fatigability over the course of the task. Perceived fatigue and performance fatigability are both relevant in many neurological diseases, potentially influence each other and share common etiologic factors [6].

The relation between perceived fatigue and performance fatigability in MS has been researched by several studies in order to achieve a better understanding of the underlying mechanisms which could lead to better targeted interventions. In addition, fatigability measurements could serve as a potential objective quantification of perceived fatigue. However, a recent meta-analysis showed significant, but weak to moderate correlations between physical fatigability and perceived fatigue in MS [7]. Similar results were found between an attentional fatigability task and perceived fatigue [8]. Overall, it is suggested that, although related and probably both relevant for MS patients, perceived fatigue and performance fatigability are different constructs and should therefore be evaluated independently [6-8]. One not yet thoroughly investigated and potential method to reliably investigate performance fatigability is the measurement of saccadic eye movements. Saccades are ballistic preprogrammed movements and the most rapid movements of the human body. They have the function of shifting the sight from one target to the next and have distinctive parameters such as peak velocity, gain and latency [9]. The control of saccades relies on complex interactions between different brain and brainstem regions and therefore even small disruptions in signal are expected to result in changes of saccadic parameters. Besides motor function, some parameters (for example latency of saccades) might also reflect cognitive functions, including processing of visual information, task planning, attention and selection of relevant stimuli [10]. Saccadic eye movements can be easily assessed by 
non-invasive infrared oculography, which results in reproducible results when using a standardized protocol [11]. There is some evidence that changes of saccadic parameters can occur over the course of a prolonged saccadic task and that saccadic parameters and changes are related to perceived fatigue $[12,13]$. In one of these studies, there was a significantly larger change in latency, amplitude and peak velocity during a 10-minutes task in MS patients with fatigue compared to non-fatigued MS patients and healthy controls [12]. Another study showed changes of saccadic parameters over time in a specific eye movement disorder in MS, internuclear ophthalmoplegia (INO) [14]. INO is a common brainstem disorder in MS $[15,16]$ and results in a delay of the adducting eye during horizontal saccades. The study showed that both substantial improvement and aggravation of the INO can occur in a saccadic fatigability task, probably related to INO severity [14]. It was suggested by the authors that INO could serve as model for studying fatigue. In both studies, the sample sizes were small and no confounding factors were investigated.

The findings in these studies lend support to further investigate changes of saccadic parameters and their relations, in a larger sample and with a standardized measurement protocol. Therefore, the purpose of this study is to (1) investigate the degree of change of saccadic parameters over time in both MS patients with and without an INO compared to healthy controls and (2) the relation between (potential changes of) saccadic parameters and perceived fatigue of these MS patients as assessed by questionnaires. We hypothesized that measurement of saccades has potential as an objective and accurate outcome measure for performance fatigability and could, more than other objective measures, reflect on perceived fatigue.

\section{Materials and Methods}

\subsection{Study design and patient population}

This study was approved by the Medical Ethical Committee on Human research of the Amsterdam UMC and has been performed in accordance with the ethical standards laid down in the 1964

Declaration of Helsinki and its later amendments. Written informed consent was obtained from all subjects before study inclusion.

For this observational cross-sectional study, MS patients and healthy controls were included from the Amsterdam MS cohort, an ongoing observational cohort of the Amsterdam UMC. Eye movement results on internuclear ophthalmoplegia and fixation abnormalities of this cohort were previously 
reported [16, 17]. Subjects were at least 18 years of age. All MS patients had to fulfil clinical and radiological criteria for a diagnosis of clinically definite MS [18]. The disease course was described as relapsing-remitting (RR), secondary progressive (SP), or primary progressive (PP) [19]. Exclusion criteria were: immunodeficiency syndrome, relapse or course of steroids within 6 weeks to inclusion, pregnancy, or history of drug or alcohol abuse.

\subsection{Clinical and ophthalmological assessment}

All assessments (clinical, infrared oculography and questionnaires) were performed on the same day and in the same order of sequence.

The disease duration was calculated in years from the first MS symptom. The Expanded Disability Status Scale (EDSS) score [20] was determined by a certified examiner to assess the level of disability. History of symptomatic MS associated optic neuritis (MSON) was based on a consensus protocol [21], including recording of the best corrected high and low contrast visual acuities (VA) using Sloan letter charts (100\% for HCVA, $2.5 \%$ for LCVA)[22]. Detecting of internuclear ophthalmoplegia (INO) was based on previously described infrared oculography criteria [16].

Perceived fatigue was assessed by two different questionnaires, the Checklist Individual Strength (CIS20R) and the Neurological Fatigue Index (NFI). The fatigue subscale of the CIS20R consists of eight items, higher scores indicate a higher degree of fatigue (range: 8-40). With this score MS patients were dichotomized into non-fatigued and fatigued (score of $>35$ for fatigued patients) $[23,24]$. The NFI is a scale specifically developed and validated for MS related fatigue, according to the strict Rasch model expectations for questionnaire development $[25,26]$. It consist of a 10-item summary scale and scales measuring the physical and cognitive components of fatigue. The ordinal raw scores of the NFI were converted into interval scale scores which can be used in parametric procedures [25]. Higher scores indicate a higher degree of fatigue and the scores range from 0 to 30 for the summary scale, 0 to 24 for the physical component and 0 to 12 for the cognitive component.

\subsection{Infrared oculography}

Data were measured and analysed using our standardised protocol suitable for a multicenter setting, the DEMoNS-protocol [11]. In brief, eye movements were measured with the Eyelink 1000 Plus eye tracker at a frequency of $1000 \mathrm{~Hz}$. Participants were seated in front of a display monitor and their head 
was stabilised by means of a chin and a forehead rest. For this study, horizontal pro-saccades of two different task were included, the first pro-saccadic task (FPT) and the repeated pro-saccadic task (RPT). Both tasks contained 30 saccades from the center of the screen to an eccentric location of 8 degrees of visual angle left or right from the center. Three other saccadic tasks (with a duration of approximately 15 minutes and including two complex tasks) were performed between FPT and RPT, and by this the protocol was designed to induce oculomotor fatigability between FPT and RPT. Therefore, the change in saccadic parameters from FPT to RPT (subtracting the individual mean values of RPT by the values of FPT), could reflect saccadic fatigability. The mean saccadic peak velocity, latency, gain, area under the curve of the saccadic trajectory (AUC) and the main sequence relation (peak velocity divided by amplitude, Pv/Am) were calculated in both tasks. For these parameters, the mean of both eyes was used.

Furthermore, the versional dysconjugacy index (VDI) of the AUC and Pv/Am were determined. The VDI is the ratio of the abducting eye value to the adducting eye value and is used for quantifying an INO $[16,27,28]$. As INO is direction dependent, the mean VDI of leftward saccades and the mean VDI of rightward saccades were investigated separately.

For the automatic and off-line analysis of the eye movement data, we used an in-house written program in Matlab (Mathworks, inc., Natick, MA) [11]. To pass quality control at least $50 \%$ of centrifugal saccades needed to be acceptable for a subject to be included.

\subsection{Statistical analyses}

Data were analysed visually and statistically for normality. Independent T-tests (Gaussian data) and non-parametric tests (non-Gaussian data) were used for the comparison of saccadic parameters between MS patients and healthy controls and between fatigued and non-fatigued MS patients (based on the CIS20R). The chi square test was used for categorical data. Linear regression analyses were used to analyse the relationship of saccadic parameters and NFI interval scores. These analyses were adjusted for the possible confounders age, sex and EDSS. When investigating fatigability parameters and relations with perceived fatigue, the MS group was primarily investigated as a whole, without exclusion of patients with specific eye movement disorders. The effect of INO on the different comparisons was investigated afterwards. 
Statistical analyses were performed using SPSS (IBM Corp. Released 2013. IBM SPSS Statistics for Windows, Version 22.0. Armonk, NY: IBM Corp.).

\section{Results}

\subsection{Study population}

In total 226 MS patients and 61 healthy controls were recruited to this study. Of these we had to exclude 35 MS patients and 3 healthy controls. The reasons for exclusion were corrupted data files $(\mathrm{N}=6)$, insufficient quality of the data $(\mathrm{N}=22)$, missing RPT task $(\mathrm{N}=9)$ and one mono-ocular measurement.

The demographic and clinical characteristics of the included subjects are summarized in table 1. In the patient group, relatively more subjects were of female gender compared to the healthy control group (69\% versus $53 \%$ respectively, $p=0.043)$. Patients had a mean disease duration of $18.4( \pm 10.0)$ years and the majority (67\%) had a RR disease course. MS patients showed significantly higher scores on the NFI summary, cognition and physical score, and CIS20R fatigue subscale than healthy controls $(p<0.001)$. 


\begin{tabular}{|c|c|c|}
\hline & $\begin{array}{l}\text { MS patients } \\
\mathrm{N}=191\end{array}$ & $\begin{array}{l}\text { Healthy controls } \\
\mathrm{N}=58\end{array}$ \\
\hline Gender (N, female) & $132(69 \%)$ & $31(53 \%)$ \\
\hline Age (years) & $53.7( \pm 10.4)$ & $52.4( \pm 9.1)$ \\
\hline Disease duration (years) & $18.4( \pm 10.0)$ & $\mathrm{N} / \mathrm{A}$ \\
\hline EDSS (median (IQR, total range)) & $\begin{array}{l}3.5(2.25 \\
0.0-8.5)\end{array}$ & N/A \\
\hline \multicolumn{3}{|l|}{ Disease type } \\
\hline RRMS (N) & $127(67 \%)$ & $N / A$ \\
\hline SPMS (N) & $48(25 \%)$ & N/A \\
\hline PPMS (N) & $13(7 \%)$ & N/A \\
\hline Unclassifiable & $3(2 \%)$ & N/A \\
\hline HCVA (mean ODS) ${ }^{a}$ & $52.9( \pm 8.8)$ & N/A \\
\hline LCVA (mean ODS) ${ }^{\mathrm{b}}$ & $29.9( \pm 11.6)$ & N/A \\
\hline Optic neuritis history $(\mathrm{N})^{\mathrm{c}}$ & $105(63 \%)$ & N/A \\
\hline Internuclear ophthalmoplegia (N) & $60(31 \%)$ & N/A \\
\hline NFI summary score & $14.7( \pm 5.5)$ & $5.9( \pm 4.3)$ \\
\hline NFI physical score & $11.8( \pm 5.0)$ & $4.3( \pm 3.3)$ \\
\hline NFI cognitive score & $5.7( \pm 2.7)$ & $2.1( \pm 2.2)$ \\
\hline CIS20R fatigue subscale & $33.2( \pm 12.8)$ & $19.3( \pm 10.3)$ \\
\hline \multicolumn{3}{|c|}{$\begin{array}{l}\text { MS: multiple sclerosis ; N: number; EDSS: expanded disability status scale; IQR: interquartile range } \\
\text { RR: relapsing-remitting; SP: secondary progressive; PP: primary progressive; HCVA: high-contrast } \\
\text { visual acuity; LCVA: low-contrast visual acuity; ODS: mean of right (OD) and left (OS) eye; NFI: } \\
\text { Neurological Fatigue Index; CIS2OR: Checklist Individual Strength } \\
\text { a: HCVA data missing from } 16 \text { patients } \\
\text { b: LCVA data missing from } 54 \text { patients } \\
\text { c: Optic neuritis information missing from } 25 \text { patients }\end{array}$} \\
\hline
\end{tabular}

Table 1 Demographic and clinical characteristics of the healthy controls and MS patients 

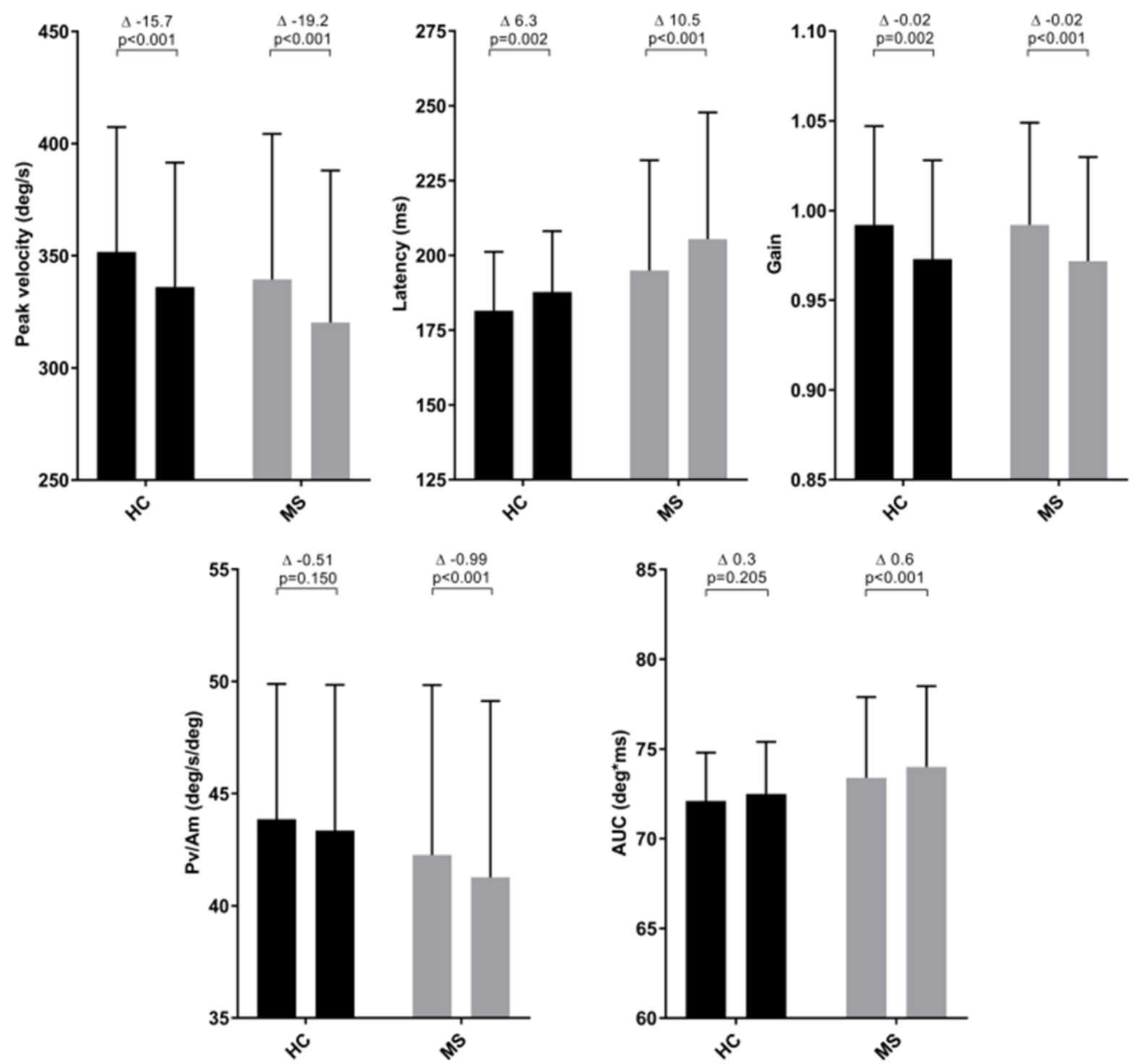

Fig 1 Saccadic parameters of healthy controls and MS patients

Bargraphs showing the mean and standard deviation (error bars) of the pro-saccadic parameters. In every graph the results of the healthy controls $(\mathrm{HC})$ are shown in black bars and the results of MS patients in grey. The left bar of both the healthy controls and MS patients represent the values of FPT, the right bar the values of RPT. At the top of the graph the change from FPT and RPT $(\Delta)$ is shown, with the corresponding $\mathrm{p}$ value. deg: degrees of visual angle; s: seconds; ms: milliseconds; Pv/Am: peak velocity divided by amplitude; AUC: Area under the curve of the horizontal saccadic trajectory 


\subsection{Comparison MS patients and healthy controls}

In table 2 and figure 1 the parameters of both tasks of the MS patients and healthy controls are summarized. In both FPT and RPT, MS patients showed a significantly longer latency compared to healthy controls (mean difference 13.5 and $17.7 \mathrm{~ms}, \mathrm{p}=0.008$ and $\mathrm{p}=0.002$, respectively). Furthermore, MS patients showed a larger AUC than healthy controls, which was for the most part accounted for by the presence of INO in the MS group (AUC FPT $71.8 \mathrm{deg}^{*} \mathrm{~ms}$ in non-INO group and 76.7 in INO group, AUC RPT 72.8 deg $^{*} \mathrm{~ms}$ in non-INO group and 76.6 in INO group). Other parameters in FPT and RPT were not significantly different between MS patients and healthy controls.

In both the MS and healthy control group, there was a significant increase in latency and decrease in peak velocity and gain from FPT to RPT. In the MS group, there was a significant decrease in PV/Am and increase in AUC from FPT to RPT (figure 1). The parameter changes from FPT to RPT were consistently higher in the MS group compared to the healthy control group, although the differences were not statistically significant (table 2). This was also the case when comparing only non-INO patients with healthy controls (data not shown).

\subsection{Relation saccadic parameters and perception based fatigue}

The results of saccadic parameters are presented in figure 2. The figure illustrates three groups, healthy controls, fatigued and non-fatigued patients with MS (based on the CIS20R). The latency and AUC of both tasks showed a significant difference between healthy controls and one or both MS groups (figure 2). Latency in both tasks was higher and peak velocity and Pv/Am slightly lower in fatigued MS patients compared to non-fatigued MS patients, although these differences were not statistically significant. Furthermore, none of the changes from FPT to RPT of the saccadic parameters was significantly different between the groups. Linear regression analyses revealed a significant relation between latency in both tasks and NFI summary score (table 3). After adjustment for EDSS (Adjusted model 2) the effect size of latency in both FPT and RPT was considerably decreased and not significant. None of the other parameters in FPT or RPT, nor the parameters changes from FPT to RPT, were significantly related to NFI summary score. 


\begin{tabular}{|c|c|c|c|c|c|c|}
\hline \multirow[t]{2}{*}{ Parameter } & \multicolumn{2}{|c|}{ MS patients } & \multicolumn{2}{|c|}{ Healthy controls } & \multicolumn{2}{|c|}{ Difference HC-MS } \\
\hline & Mean & $S D$ & Mean & $S D$ & $\begin{array}{l}\text { Mean } \\
(95 \% \mathrm{Cl})\end{array}$ & $P$ value \\
\hline $\begin{array}{l}\text { FPT Peak velocity } \\
\text { (deg/s) }\end{array}$ & 339.4 & 64.9 & 351.7 & 55.7 & $\begin{array}{c}12.3 \\
(-6.3-30.8) \\
\end{array}$ & 0.195 \\
\hline $\begin{array}{l}\text { RPT Peak velocity } \\
\text { (deg/s) }\end{array}$ & 320.2 & 67.8 & 336.0 & 55.6 & $\begin{array}{c}15.8 \\
(-3.4-35.0)\end{array}$ & 0.107 \\
\hline $\begin{array}{l}\Delta \text { Peak velocity } \\
(\mathrm{deg} / \mathrm{s})\end{array}$ & -19.2 & 21.4 & -15.7 & 25.6 & $\begin{array}{c}3.5 \\
(-3.1-10.2) \\
\end{array}$ & 0.296 \\
\hline FPT Latency (ms) & 194.9 & 36.9 & 181.4 & 19.8 & $\begin{array}{c}-13.5 \\
(-23.4--3.5)\end{array}$ & 0.008 \\
\hline RPT Latency (ms) & 205.5 & 42.3 & 187.7 & 20.4 & $\begin{array}{c}-17.7 \\
(-29.1--6.4)\end{array}$ & 0.002 \\
\hline$\Delta$ Latency $(\mathrm{ms})$ & 10.5 & 21.1 & 6.3 & 15.0 & $\begin{array}{c}-4.2 \\
(-10.1-1.6) \\
\end{array}$ & 0.155 \\
\hline FPT Gain & 0.99 & 0.06 & 0.99 & 0.06 & $\begin{array}{c}-0.00 \\
(-0.02-0.01)\end{array}$ & 0.989 \\
\hline RPT Gain & 0.97 & 0.05 & 0.97 & 0.05 & $\begin{array}{c}0.00 \\
(-0.02-0.02) \\
\end{array}$ & 0.886 \\
\hline$\Delta$ Gain & -0.02 & 0.04 & -0.02 & 0.04 & $\begin{array}{c}0.00 \\
(-0.01-0.01)\end{array}$ & 0.817 \\
\hline $\begin{array}{l}\text { FPT Pv/Am } \\
\text { (deg/s/deg) }\end{array}$ & 42.3 & 7.6 & 43.9 & 6.0 & $\begin{array}{c}1.6 \\
(-0.5-3.7) \\
\end{array}$ & 0.143 \\
\hline $\begin{array}{l}\text { RPT Pv/Am } \\
\text { (deg/s/deg) }\end{array}$ & 41.3 & 7.9 & 43.4 & 6.5 & $\begin{array}{c}2.1 \\
(-0.2-4.3) \\
\end{array}$ & 0.068 \\
\hline $\begin{array}{l}\Delta \mathrm{Pv} / \mathrm{Am} \\
\text { (deg/s/deg) }\end{array}$ & -1.0 & 2.4 & -0.5 & 2.7 & $\begin{array}{c}0.5 \\
(-0.2-1.2) \\
\end{array}$ & 0.188 \\
\hline FPT AUC (deg*ms) & 73.4 & 4.5 & 72.1 & 2.7 & $\begin{array}{c}-1.3 \\
(-2.5--0.03)\end{array}$ & 0.044 \\
\hline RPT AUC (deg*ms) & 74.0 & 4.5 & 72.5 & 2.9 & $\begin{array}{c}-1.5 \\
(-2.8--0.27)\end{array}$ & 0.017 \\
\hline$\triangle \mathrm{AUC}\left(\mathrm{deg}^{*} \mathrm{~ms}\right)$ & 0.6 & 2.2 & 0.3 & 1.9 & $\begin{array}{c}-0.3 \\
(-0.9-0.37)\end{array}$ & 0.409 \\
\hline $\begin{array}{l}\text { MS: multiple sclerosis; } \\
\text { saccadic task; RPT: repe } \\
\text { seconds; ms: millisecon } \\
\text { horizontal saccadic traje }\end{array}$ & $\begin{array}{l}\text { healthy c } \\
\text { d pro-sac } \\
\text { Pv/Am: p } \\
\text { ry }\end{array}$ & $\begin{array}{l}\text { rols; SD } \\
\text { dic task } \\
\text { k velocit }\end{array}$ & $\begin{array}{l}\text { ard deviat } \\
\text { ue of RPT } \\
\text { d by amp }\end{array}$ & $\begin{array}{l}\text { Cl: conf } \\
\text { us FPT; } \\
\text { le; AUC: }\end{array}$ & $\begin{array}{l}\text { e interval; FPT: fir } \\
\text { degrees of visual a } \\
\text { under the curve of }\end{array}$ & $\begin{array}{l}\text { pro- } \\
\text { le; s: } \\
\text { le }\end{array}$ \\
\hline
\end{tabular}

Table 2 Results of both pro-saccadic tasks, comparing MS patients with healthy controls 

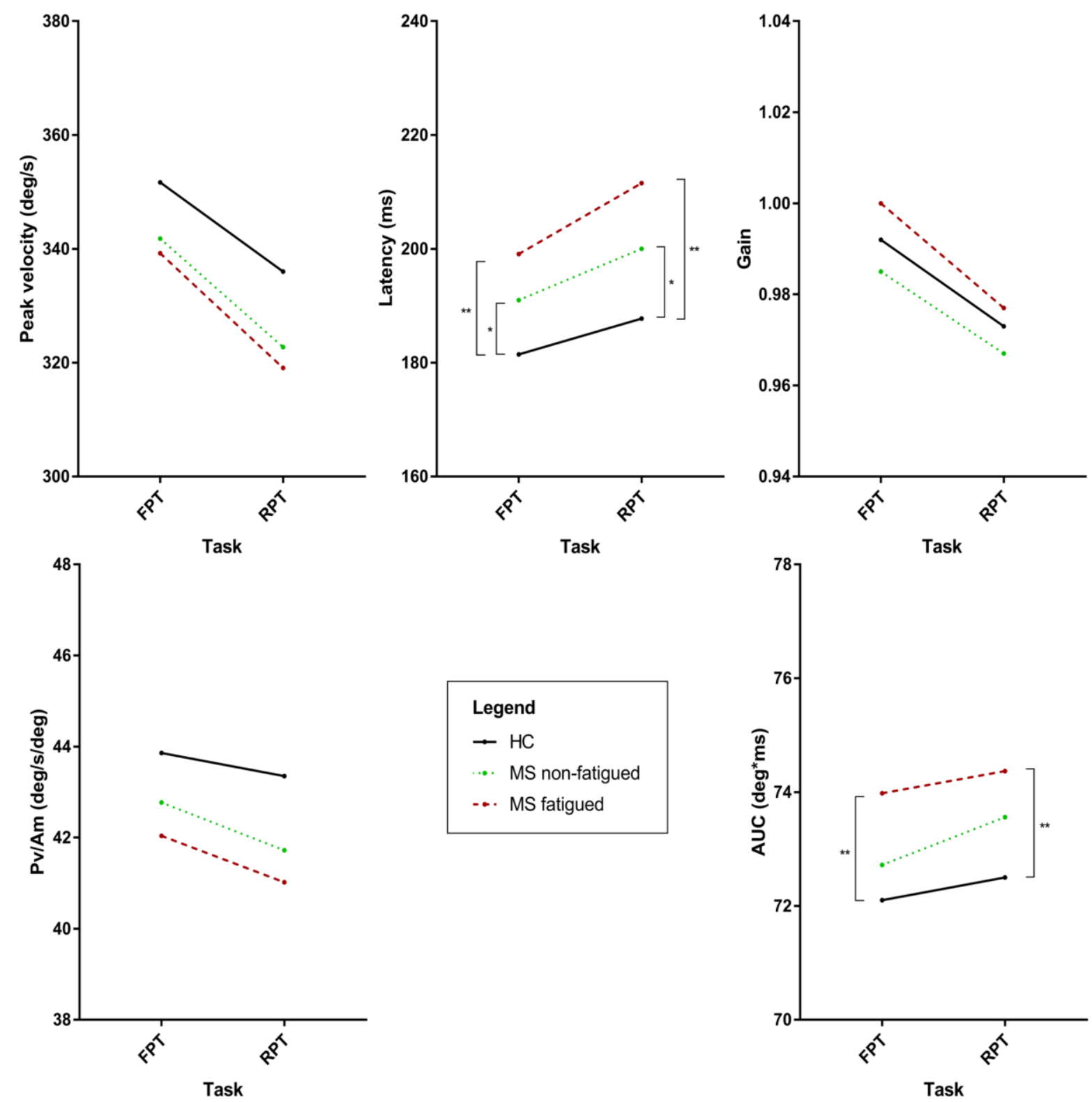

Fig 2 Saccadic parameters of healthy controls, fatigued and non-fatigued MS patients

Linegraphs showing the mean and change from FPT to RPT of the pro-saccadic parameters, separately for healthy controls (HC, solid line), non-fatigued MS patients (dotted line) and fatigued MS patients (dashed line). deg: degrees of visual angle; s: seconds; ms: milliseconds; Pv/Am: peak velocity divided by amplitude; AUC: Area under the curve of the horizontal saccadic trajectory; * : $p<0.05 ;{ }^{* *}: p<0.01$ 


\begin{tabular}{lllll}
\hline Parameter & Linear regression & $\boldsymbol{\beta}$ & $\mathbf{9 5 \%} \mathbf{C l}$ & $\boldsymbol{p}$ value \\
\hline \multirow{3}{*}{ FPT Latency (ms) } & Crude & 0.022 & $0.000-0.044$ & 0.049 \\
& Adjusted model 1 & 0.019 & $-0.003-0.042$ & 0.089 \\
& Adjusted model 2 & -0.001 & $-0.023-0.022$ & 0.933 \\
\hline \multirow{2}{*}{ RPT Latency (ms) } & Crude & 0.023 & $0.003-0.042$ & 0.021 \\
& Adjusted model 1 & 0.020 & $0.001-0.039$ & 0.008 \\
& Adjusted model 2 & 0.003 & $-0.017-0.022$ & 0.787 \\
\hline \multirow{3}{*}{$\begin{array}{l}\text { Latency (ms) } \\
\text { Crude }\end{array}$} & Adjusted model 1 & 0.023 & $-0.015-0.061$ & 0.232 \\
& Adjusted model 2 & 0.012 & $-0.017-0.059$ & 0.282 \\
\hline Crude: unadjusted model; Adjusted model 1: adjustment for sex and disease duration; Adjusted model 2: adjustment \\
for sex, disease duration, EDSS
\end{tabular}

Table 3 Linear regression analysis demonstrating the association between latency parameters and NFI summary score

\subsection{Fatigability of INO}

As demonstrated before [16] VDI AUC and Pv/Am values of non-INO MS patients and healthy controls lied just above value one, as on average the abducting eye is slightly faster than the adducting eye. INO patients showed a mean VDI AUC of $1.44 \pm 0.27$ and a mean VDI PV/Am of $1.46 \pm 0.52$ (mean of both tasks and both directions). In figure 3 Bland-Altman plots are illustrating the change of VDI AUC from FPT to RPT against the mean VDI AUC of INO and non-INO patients. On average, there was no statistical significant change in VDI values from FPT to RPT in both groups. The $95 \%$ limits of agreement (95\% confidence interval of the mean difference) were considerably further apart in the INO group than the non-INO group (-0.09 to 0.11 in the non-INO group and -0.24 to 0.32 in INO group, mean of both directions). This indicates that an INO can both improve and aggravate during a fatigability task. The change of VDI AUC values in the non-INO group was comparable to the healthy control group (mean difference $0.01(95 \% \mathrm{Cl}-0.11$ to 0.14$)$ in the healthy control group, mean of both directions). There was no considerable proportional bias in the INO group (slope of regression line 0.001 for leftward INO and 0.110 for rightward INO). Finally, no significant relation between change of the VDI values in the INO group and perceived fatigue (both CIS20R and NFI questionnaire) was found. 

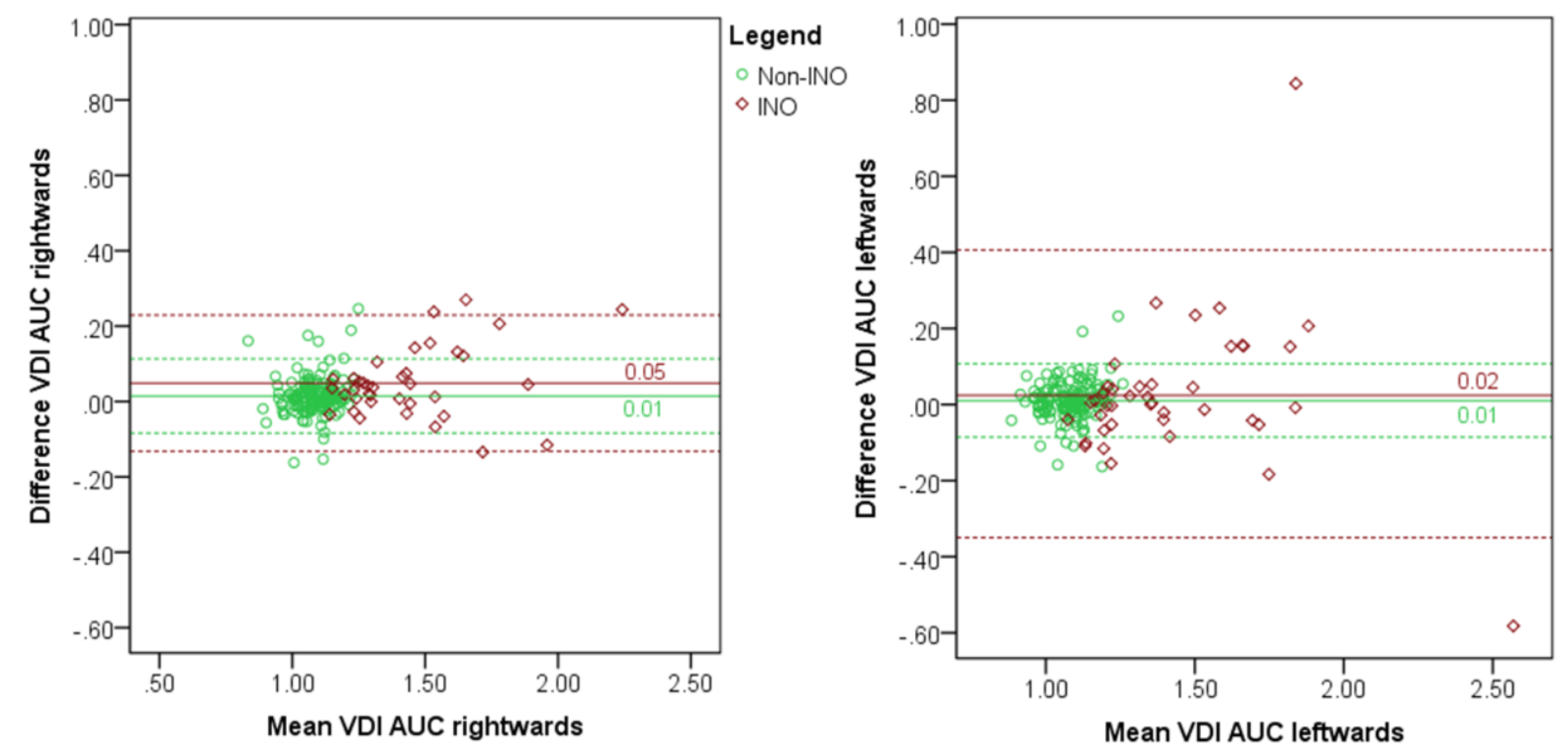

Fig 3 Bland-Altman plots of the VDI AUC

Bland-Altman plots showing the change from FPT to RPT against the mean of FPT and RPT of the VDI AUC. The left graph shows the VDI AUC of rightward saccades, the right graphs the VDI AUC of leftward saccades. The dots represent the MS patients without an INO and in the diamonds MS patients with an INO. The mean difference of both groups is indicated with the solid lines and the corresponding $95 \%$ confidence interval with the dashed lines

\subsection{Discussion}

To our knowledge, this is the first study that systematically investigated changes of saccadic parameters in a large cohort. This study showed that with this method saccadic fatigability can be evoked in MS patients and healthy controls. This fatigability was not decisively MS related, although the differences between FPT and RPT in our study were more pronounced in the MS group than in the healthy control group. There were no clear relations found between saccadic fatigability and perceived fatigue in the MS group. Furthermore, the significant relation between latency and perceived fatigue had a small effect size and was (largely) explained by the disability status of the patients.

Eye movement measurement in the current study was performed with a modern, high-frequency and non-invasive eye tracking device. The protocols for measurement and analysis are standardized and open-source available. Furthermore, parameters showed high reproducibility [11]. Altogether, this 
makes the eye movement measurement reliable, transparent for other researchers and suitable for a multi-center setting. Therefore, the prognostic and diagnostic value of saccadic fatigability could be tested in conditions such as ocular myasthenia gravis, thyroid eye disease and other myopathic conditions of the extraocular muscles.

Previous studies with smaller sample sizes showed significant relations between (changes in) saccadic parameters and perceived fatigue $[12,13]$. Protocols differed from the protocol in the current study. Amongst others, saccadic fatigability was assessed with one prolonged task and larger target amplitudes were used. Theoretically, the relation between saccadic fatigability and perceived fatigue in our study could be underestimated by these differences. However, considering the demonstrated saccadic fatigability in the MS patients and the sample size of our study, these relations are not expected to exist in our sample. This confirms, as shown in previous studies $[7,8]$, that there is no simple relationship between fatigability and subjective fatigue perception.

The findings on fatigability of INO are in line with literature [14]. Both aggravation and improvement of the INO can occur in a fatigability task. Matta et al. proposed that improvement of conjugacy is mediated by the recruitment of vergence movements [14]. These movements are relatively spared in INO and this theory was supported by phase-plane plots of the saccadic eye movements. Improvement of conjugacy in their study was observed in patients with a more severe degree of INO, which suggested that possible central adaptive mechanisms may have developed in these INO patients, who might have a longer duration of the INO. Improvement in more severe INO's in comparison to less severe INO's was not observed in our study. However, due to the cross sectional nature of our study, we do not have information on the duration of the INO. Testing fatigability of INO in a longitudinal design and in treatment trials might help to reveal underlying mechanisms and characteristics of MS patients that either show improvement or aggravation of their INO in a fatigability task. In this way the extent of clinical and scientific applications of testing fatigability of INO can be investigated.

The influence of disability status of MS patients on the relation between saccadic parameters and fatigue can be put in context of previous literature. Although some conflicting results exist, several studies showed relations between fatigue and general disability [5, 29-31]. Similar, relations between ocular motor disorders and general disability in MS patients were found $[32,33]$. However, only clinical examinations of ocular motor disorders have been used in these studies and the relation with deficits in different disease domains is unknown. 
Investigating quantified saccadic parameters in relation to disease characteristics, as physical and cognitive disability and structural damage, will be part of future work.

\subsection{Conclusion}

In this study we showed that saccadic fatigability can be demonstrated, but is of no added value in MS. Furthermore, there was a lack of a strong relation between saccadic fatigability and perception based fatigue. Third, a large influence of disability status on relations between saccadic parameters and fatigue was found. Altogether, this suggest that saccadic testing is not perfectly suitable as a measure for objective quantification of MS related fatigue, certainly not on an individual patient level. To generalize this for MS patient care and future MS studies, we advise to consider objective fatigability and subjective fatigue in MS as different concepts. Finally, we provided an objective and reliable method which has the potential to be of diagnostic and prognostic value in disorders of the neuro-muscular junction and muscles of the eye.

The measurement and analysis protocol is available on:

https://www.protocols.io/view/demons-protocol-for-measurement-and-analysis-of-ey-ruad6se 


\section{References}

[1] R. Bakshi, Z.A. Shaikh, R.S. Miletich, D. Czarnecki, J. Dmochowski, K. Henschel, V. Janardhan, N.

Dubey, P.R. Kinkel, Fatigue in multiple sclerosis and its relationship to depression and neurologic disability, Mult Scler 6(3) (2000) 181-5.

[2] R. Bakshi, Fatigue associated with multiple sclerosis: diagnosis, impact and management, Mult Scler 9 (2003) 219-227.

[3] T.J. Weiland, G.A. Jelinek, C.H. Marck, E.J. Hadgkiss, D.M. van der Meer, N.G. Pereira, K.L. Taylor, Clinically significant fatigue: prevalence and associated factors in an international sample of adults with multiple sclerosis recruited via the internet, PLoS One 10(2) (2015) e0115541.

[4] R. Bergamaschi, A. Romani, M. Versino, R. Poli, V. Cosi, Clinical aspects of fatigue in multiple sclerosis, Funct Neurol 12(5) (1997) 247-51.

[5] P. Flachenecker, T. Kumpfel, B. Kallmann, M. Gottschalk, O. Grauer, P. Rieckmann, C.

Trenkwalder, K.V. Toyka, Fatigue in multiple sclerosis: a comparison of different rating scales and correlation to clinical parameters, Mult Scler 8(6) (2002) 523-6.

[6] B.M. Kluger, L.B. Krupp, R.M. Enoka, Fatigue and fatigability in neurologic illnesses: proposal for a unified taxonomy, Neurology 80(4) (2013) 409-16.

[7] B.D. Loy, R.L. Taylor, B.W. Fling, F.B. Horak, Relationship between perceived fatigue and performance fatigability in people with multiple sclerosis: A systematic review and meta-analysis, $\mathrm{J}$ Psychosom Res 100 (2017) 1-7.

[8] M. Aldughmi, J. Bruce, C.F. Siengsukon, Relationship Between Fatigability and Perceived Fatigue Measured Using the Neurological Fatigue Index in People with Multiple Sclerosis, Int J MS Care 19(5) (2017) 232-239.

[9] R.J. Leigh, D.S. Zee, The saccadic system, The neurology of eye movements, Oxford University Press, Oxford, 2015, pp. 169-251.

[10] R.J. Leigh, D.S. Zee, The neurology of eye movements, 5 ed., Oxford University Press, Oxford, 2015.

[11] J.A. Nij Bijvank, A. Petzold, L.J. Balk, H.S. Tan, B.M.J. Uitdehaag, M. Theodorou, L.J. van Rijn, A standardized protocol for quantification of saccadic eye movements: DEMoNS, PLoS ONE 13(7) (2018) e0200695. 
[12] C. Finke, L.M. Pech, C. Sommer, J. Schlichting, S. Stricker, M. Endres, F. Ostendorf, C.J. Ploner, A.U. Brandt, F. Paul, Dynamics of saccade parameters in multiple sclerosis patients with fatigue, $\mathrm{J}$ Neurol 259(12) (2012) 2656-63.

[13] M. Ferreira, P.A. Pereira, M. Parreira, I. Sousa, J. Figueiredo, J.J. Cerqueira, A.F. Macedo, Using endogenous saccades to characterize fatigue in multiple sclerosis, Mult Scler Relat Disord 14 (2017) 16-22.

[14] M. Matta, R.J. Leigh, M. Pugliatti, I. Aiello, A. Serra, Using fast eye movements to study fatigue in multiple sclerosis, Neurology 73 (2009) 798-804.

[15] M. Jozefowicz-Korczynska, M. Lukomski, A. Pajor, Identification of internuclear ophthalmoplegia signs in multiple sclerosis patients. Saccade test analysis, J Neurol 255(7) (2008) 1006-11.

[16] J.A. Nij Bijvank, L.J. Rijn, L.J. Balk, H.S. Tan, B.M.J. Uitdehaag, A. Petzold, Diagnosing and quantifying a common deficit in multiple sclerosis: internuclear ophthalmoplegia, Neurology (2019 Apr 19 [Epub ahead of print]).

[17] J.A. Nij Bijvank, A. Petzold, D. Coric, H.S. Tan, B.M.J. Uitdehaag, L.J. Balk, L.J. van Rijn, Quantification of Visual Fixation in Multiple Sclerosis, Invest Ophthalmol Vis Sci 60(5) (2019) 13721383.

[18] C.H. Polman, S.C. Reingold, B. Banwell, M. Clanet, J.A. Cohen, M. Filippi, K. Fujihara, E. Havrdova, M. Hutchinson, L. Kappos, F.D. Lublin, X. Montalban, P. O'Connor, M. Sandberg-Wollheim, A.J. Thompson, E. Waubant, B. Weinshenker, J.S. Wolinksky, Diagnostic criteria for multiple sclerosis: 2010 revisions to the McDonald criteria, Ann Neurol 69(2) (2011) 293-302.

[19] F.D. Lublin, S.C. Reingold, Defining the clinical course of multiple sclerosis: Results of an international survey. National Multiple Sclerosis Society (USA) Advisory Committee on Clinical Trials of New Agents in Multiple Sclerosis, Neurology 46 (1996) 907-911.

[20] J.F. Kurtzke, Rating neurological impairment in multiple sclerosis: An expanded disability status scale (EDSS), Neurology 33 (1983) 1444-1452.

[21] A. Petzold, M.P. Wattjes, F. Costello, J. Flores-Rivera, C.L. Fraser, K. Fujihara, J. Leavitt, R. Marignier, F. Paul, S. Schippling, C. Sindic, P. Villoslada, B. Weinshenker, G.T. Plant, The investigation of acute optic neuritis: a review and proposed protocol, Nat Rev Neurol 10(8) (2014) 44758. 
[22] L.J. Balcer, M.L. Baier, J.A. Cohen, M.F. Kooijmans, A.W. Sandrock, M.L. Nano-Schiavi, D.C. Pfohl, M. Mills, J. Bowen, C. Ford, F.R. Heidenreich, D.A. Jacobs, C.E. Markowitz, W.H. Stuart, G.S. Ying, S.L. Galetta, M.G. Maguire, G.R. Cutter, Contrast letter acuity as a visual component for the Multiple Sclerosis Functional Composite, Neurology 61 (2003) 1367-1373.

[23] J.H. Vercoulen, C.M. Swanink, J.F. Fennis, J.M. Galama, J.W. van der Meer, G. Bleijenberg, Dimensional assessment of chronic fatigue syndrome, J Psychosom Res 38(5) (1994) 383-92.

[24] M. Worm-Smeitink, M. Gielissen, L. Bloot, H.W.M. van Laarhoven, B.G.M. van Engelen, P. van Riel, G. Bleijenberg, S. Nikolaus, H. Knoop, The assessment of fatigue: Psychometric qualities and norms for the Checklist individual strength, J Psychosom Res 98 (2017) 40-46.

[25] R.J. Mills, C.A. Young, J.F. Pallant, A. Tennant, Development of a patient reported outcome scale for fatigue in multiple sclerosis: The Neurological Fatigue Index (NFI-MS), Health Qual Life Outcomes $8(2010) 22$.

[26] R.J. Mills, M. Calabresi, A. Tennant, C.A. Young, Perceived changes and minimum clinically important difference of the Neurological Fatigue Index for multiple sclerosis (NFI-MS), Mult Scler 19(4) (2013) 502-5.

[27] J.P. Flipse, C.S.M. Straathof, J. van der Steen, A.F. van Leeuwen, P.A. van Doorn, F.G.A. van der Meche, H. Collewijn, Binocular saccadic eye movements in multiple sclerosis, Journal of Neurological Sciences 148 (1997) 53-65.

[28] E.M. Frohman, T.C. Frohman, P. O'Suilleabhain, H. Zhang, K. Hawker, M.K. Racke, W. Frawley, J.T. Phillips, P.D. Kramer, Quantitative oculographic characterisation of internuclear ophthalmoparesis in multiple sclerosis: the versional dysconjugacy index Z score, J Neurol Neurosurg Psychiatry 73 (2002) 51-55.

[29] E. Patrick, C. Christodoulou, L.B. Krupp, Longitudinal correlates of fatigue in multiple sclerosis, Mult Scler 15 (2009) 258-261.

[30] D.C. Kroencke, S.G. Lynch, D.R. Denney, Fatigue in multiple sclerosis: relationship to depression, disability, and disease pattern, Mult Scler 6(2) (2000) 131-6.

[31] S. Kaya Aygunoglu, A. Celebi, N. Vardar, E. Gursoy, Correlation of Fatigue with Depression, Disability Level and Quality of Life in Patients with Multiple Sclerosis, Noro Psikiyatr Ars 52(3) (2015) 247-251. 
[32] G. Servillo, D. Renard, G. Taieb, P. Labauge, S. Bastide, M. Zorzon, G. Castelnovo, Bedside tested ocular motor disorders in multiple sclerosis patients, Mult Scler Int 2014 (2014) 732329.

[33] J. Derwenskus, J.C. Rucker, A. Serra, J.S. Stahl, D.L. Downey, N.L. Adams, R.J. Leigh, Abnormal eye movements predict disability in MS: two-year follow-up, Ann N Y Acad Sci 1039 (2005) 521-3. 